
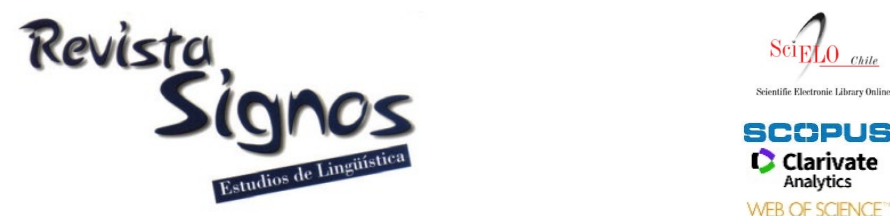

\title{
Agentividad y discurso. La proyección discursiva de los procesos
}

Agentivity and discourse. Discursive projection of processes

\author{
Salvio Martín Menéndez \\ UNIVERSIDAD DE BUENOS AIRES \\ UNIVERSIDAD NACIONAL DE MAR DEL PLATA \\ CONICET \\ ARGENTINA \\ salviomenendez@gmail.com
}

Recibido: 24-IX-2019 / Aceptado: 28-VIII-2020

DOI: $10.4067 / \mathrm{S} 0718-09342021000100214$

\section{Resumen}

El objetivo del presente trabajo es demostrar que la organización gramatical de los rasgos que permiten caracterizar los procesos (realizados por las bases lexicales de los verbos) depende, en última instancia, de la organización estratégica de los discursos. Esto permite explicar el funcionamiento efectivo de los recursos gramaticales en relación con las estrategias de los que son parte constitutiva. Sostenemos, entonces, en principio que hay una organización jerárquica potencial de rasgos semánticos que son los que aparecen codificados en la gramática y una realización texto-discursiva específica que depende de cómo esa jerarquía se realiza efectivamente en los textos y forma parte de los recursos de las estrategias discursivas que el sujeto discursivo pone en funcionamiento cuando interactúa discursivamente. Para eso es necesario explicar el alcance de la agentividad de los procesos en dos planos co-ocurrentes y complementarios: el gramatical y el discursivo. Por lo tanto, postulamos un principio de gradualidad de base semántico-gramatical cuya explicación e interpretación depende de su inscripción semántico-discursiva. En última instancia, la finalidad del planteo es poder proveer una explicación del uso del proceso (base léxica del verbo) como uno de los recursos que componen las estrategias discursivas. Mostraremos la efectividad de nuestra propuesta a partir del análisis de una serie discursiva compuesta por titulares de diarios sobre una noticia particular.

Palabras Clave: Verbo, recurso, grado, agentividad, estrategia. 


\begin{abstract}
The objective of this paper is to demonstrate that the grammatical organization of the features that allow characterizing the processes (performed by the lexical bases of the verbs) depends, ultimately, on the strategic organization of discourse. This allows explaining the effective functioning of grammar resources in relation to the strategies of which they are a constitutive part. We hold, then, in principle that there is a potential hierarchical organization of semantic features that appear coded in grammar and a specific text-discursive realization that depends on how that hierarchy is effectively realized in the texts and is part of the resources of the discursive strategies that the discursive subject puts into action when interaction takes place. For that, it is necessary to explain the scope of agentivity of the processes in two co-occurring and complementary planes: grammatical and discursive. Therefore, we postulate a principle of semantic-grammar-based gradualness whose explanation and interpretation depends on its semantic-discursive inscription. Ultimately, the purpose of the statement is to be able to provide an explanation of the use of the process (lexical basis of the verb) as one of the resources that make up the discursive strategies. We will show the effectiveness of our proposal from the analysis of a discursive series composed of newspaper headlines about a specific news.
\end{abstract}

Key Words: Verb, resource, degree, agentivity, strategy.

\title{
INTRODUCCIÓN
}

La agentividad es un tópico que permite plantear un importante problema que toda teoría lingüística enfrenta: la relación entre gramática y discurso.

Los enfoques discursivos de base funcional (Lavandera, 1985, 1988, 2014; Menéndez, 2005a, 2014) pusieron en conflicto la visión formalista del lenguaje a partir de cuestionar los límites de las diferentes unidades de análisis que los representan. Esto supone considerar si su naturaleza es discreta o continua (y, en consecuencia, gradual).

Esquemáticamente, podría plantearse que los análisis formales consideran mayoritariamente que las unidades lingüísticas son discretas, mientras que los análisis discursivos tienden a aceptarlas como graduales. Pero esta simplificación no permite una explicación satisfactoria del fenómeno en cuestión. La razón es simple: esto no es así en sentido estricto. Tanto gramáticas de base estructural (Tesniere, 1958) como generativas (Fillmore, 1968) pusieron en cuestionamiento la absoluta centralidad formal sobre la semántica. En consonancia con estos planteos, y desde un enfoque basado en una perspectiva funcional, Halliday (1967a,1967b, 1968) intenta plantear soluciones coincidentes. Si bien los objetos de estudio en los tres casos son diferentes (con sus consecuencias metodológicas y analíticas), la base semántico-discursiva aparece como una necesidad para el planteo gramatical que permite establecer las bases para su proyección y análisis discursivo posterior. La interdependencia entre gramática y discurso es evidente; de ahí la necesidad de que la teoría gramatical esté orientada discursivamente. Pensar la gramática funcionalmente supone entenderla 
como una teoría de la gramática orientada hacia la semántica discursiva (Halliday, 1978, 1985, 2013).

La perspectiva socio-cognitivo-cultural (Halliday \& Matthiessen, 1999, 2014) de la gramática sistémico-funcional (GSF, de aquí en más) plantea que el lenguaje es uno de los sistemas semióticos a partir del que construimos nuestra visión del mundo. En ese marco general, la lingüística sistémico-funcional (LSF, de aquí en más) toma en consideración el lenguaje como un potencial de significado que se describe a partir de una red de paradigmas disponibles (una paradigmática) que el hablante pone en funcionamiento cuando opta (una sintagmática). La opción, concepto central de la LSF (Halliday, 1977, 1978) supone seleccionar diferentes componentes de esos paradigmas para que se realicen en los textos concretos inscriptos en registros (Halliday, 1978) y géneros particulares (Bajtin, 1984; Martin, 1984, 1992, 2012, 2013; Martin \& Rose, 2008).

La GSF (Halliday \& Matthiessen, 2014) reconoce tres funciones; la ideativa, la interpersonal y la textual. Cada una de ellas focaliza un aspecto del significado y está realizada por un sistema particular: el sistema de transitividad, el de tema y el de modo. Cada uno de ellos cubre una esfera del significado que tiene como punto de organización estructural la cláusula -lugar en el que se proyectan las tres funciones del lenguaje-, que se realiza cohesivamente en el texto (Halliday \& Hasan, 1976) y se proyecta estratégicamente en el discurso (Menéndez, 2005a, 2010, 2011a, 2011b). Sistema, estructura, cohesión y estrategia son cuatro conceptos centrales que se presuponen mutuamente y permiten dar cuenta de la compleja relación entre cognición, discurso y sociedad.

El objeto del presente trabajo es demostrar que la organización gramatical de los rasgos que permiten caracterizar los procesos (realizados por las bases lexicales de los verbos) depende, en última instancia, de la organización estratégica de los discursos (Menéndez, 2005a, 2005b). Esto permite explicar el funcionamiento efectivo de los recursos gramaticales en relación con las estrategias de los que son parte constitutiva.

Sostenemos, entonces, en principio que hay: i) una organización jerárquica potencial de rasgos semánticos, que aparecen codificados en la gramática; ii) una realización texto-discursiva específica, que depende de cómo esa jerarquía se realiza efectivamente en los textos, y forma parte de los recursos de las estrategias discursivas que el sujeto discursivo pone en funcionamiento cuando interactúa discursivamente. Para eso es necesario explicar el alcance de la agentividad de los procesos en dos planos co-ocurrentes y complementarios: el gramatical y el discursivo. Por lo tanto, postulamos un principio de gradualidad de base semántico-gramatical (Menéndez, 2013) cuya explicación e interpretación depende de su inscripción semánticodiscursiva. 
En última instancia, la finalidad del planteo es poder proveer una explicación del uso del proceso (base léxica del verbo) como uno de los recursos que componen las estrategias discursivas (Menéndez, 2000, 2005b).

\section{El significado ideativo}

La discrecionalidad es una propiedad formal que permite establecer oposiciones. Es la base de la denominada lingüística moderna en general y de las teorías formales, en particular. La continuidad es una propiedad no opositiva sino gradual. Es la base de las teorías funcionales de base semántico-discursiva o pragmática.

De los tres sistemas que realizan las tres funciones del lenguaje en la cláusula, solo la transitividad reconoce una naturaleza estrictamente continua ya que se realiza en la relación semántica entre la raíz verbal de los procesos y los roles exigidos por ella. Si bien puede sostenerse que la discrecionalidad aparece en la oposición base léxica del proceso y roles exigidos, la extensión semántica de la base léxica permite sostener que hay continuidad semántica que tiene su punto de inicio en la gramática y de culminación en el discurso.

El significado ideativo es un significado que se asocia al contenido discursivo. Tradicionalmente, se lo caracteriza como el significado de lo que se dice en relación con cómo se dice y la organización de la información que conlleva.

Se realiza léxico-gramaticalmente en las raíces verbales y la extensión semántica que estas conllevan en relación con los roles que se proyectan en la cláusula y en el texto. Uno de los obstáculos centrales con los que uno se enfrenta cuando trabaja con los roles es cuál es su organización semántica de base. Esto puede traducirse a partir de proponer organizar tipologías en los que se identifican los diferentes significados ideativos en grupos que sean, más o menos, representativos.

Para organizar el continum semántico de alguna manera, la LSF, propone, en su versión tradicional (Halliday \& Matthiessen, 2014), una tipología organizada de la siguiente manera: se parte de un triángulo cuyos tres vértices representan lo que Halliday denomina el mundo de las relaciones abstractas, de las relaciones físicas y de la relaciones de la consciencia. Estas serían las tres zonas semánticas sobre las que uno podría ubicar el conjunto de las bases léxicas de los verbos. Sería el mundo del ser, del hacer y del percibir. A partir de ahí, tendríamos una primera tripartición: los procesos relacionales, los procesos materiales y los procesos mentales-sensoriales. Como esto es un continum, podemos tener verbos en los que tengamos rasgos compartidos de dos de los tres subtipos principales. Entonces, tenemos los verbos de conducta, los procesos verbales y los procesos existenciales. Los relacionales nos van a permitir dar atributo, identidad y simbolizar; los materiales nos van a dar ámbitos como el suceder, el crear o cambiar, el hacer o actuar; pensar, sentir y percibir serían los verbos que 
cubrirían el aspecto mental; dentro de los de conducta, comportarse; dentro de los verbales, el decir; dentro de los existenciales, el existir. Los verbos existenciales tienen componentes relacionales y materiales, los verbales tienen componentes relacionales y mentales, y los verbos de conducta tienen componentes mentales y materiales. Es decir, lo que estamos viendo es, en realidad, una continuidad en la que pueden establecerse grados. Si bien la determinación parece intentar un planteo discreto, la postura que se adopta es la de una gradualidad que va de lo más concreto a lo más abstracto (o vice-versa). Esquemáticamente:

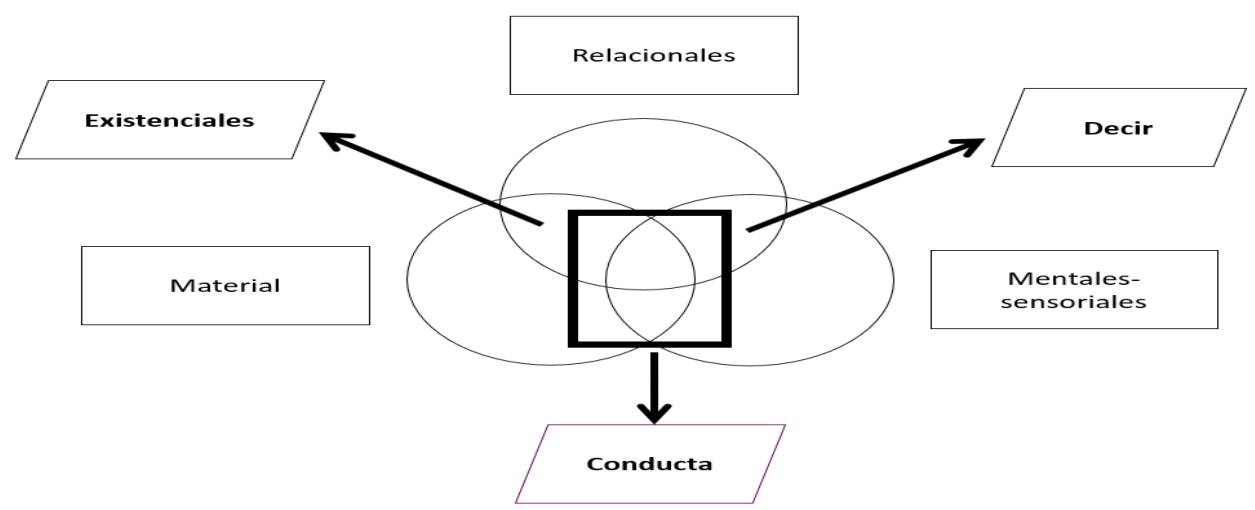

Figura 1. Tipología verbal clásica (Halliday \& Matthiessen, 2014).

El problema de las tipologías de base semántica es establecer tipos, que son entidades discretas, a partir de rasgos que son continuos. El punto básico es simple y reside en que se puede pensar en que la semántica ideativa es opositiva cuando, en realidad, es gradual.

El tipo tiene una base discreta; de ahí que su organización parece siempre apuntar a la oposición más allá de que apunte a la especificación (delicacy) (Halliday, 1967a, 1967b, 1968; Hasan, 1987; Halliday \& Matthiessen, 1999, 2014). De algún modo, este concepto apunta, justamente, en la dirección de nuestro planteo: analizar los grados de especificidad de un determinado contenido semántico.

Esto constituye, sin duda, un problema central para nuestro objetivo. De ahí que postularemos una reformulación operativa en función del análisis estratégico que postulamos. Sostenemos, por lo tanto, que la naturaleza continua del significado de los procesos realizada por las raíces verbales asociadas con los roles que exigen nos llevan a postular que no hay, en principio, procesos básicos y derivados.

Consideramos que todo proceso es un conjunto de rasgos cuya organización jerárquica reconoce dos planos: uno potencial, inscripto en la gramática y otro efectivo, realizado discursivamente y enmarcado por el registro y el género. Esa inscripción es la que nos permite encontrar la proyección discursiva de los procesos. 
Partimos para eso del principio de gradualidad que, desde un punto de vista semántico-discursivo (Verschueren, 1999; Menéndez, 2005a), permite establecer una relación entre gramática, discurso y contexto.

La opción seleccionada se realiza discursivamente a partir de un doble condicionamiento: el del registro, de manera inmediata; y el del género, de manera mediata. Este condicionamiento es probabilístico: es esperable que suceda (Matthiessen, 2015).

Para poder dar cuenta de cómo opera la relación entre gramática y discurso, se debe partir de tres variables que permiten determinar la jerarquía de los rasgos. Estas son: 1) el orden jerárquico de los rasgos, 2) el grado de concreción del proceso y 3) la relación entre causa y ejecución (Menéndez, 2017a, 2017b).

\subsection{El orden de los rasgos}

Partimos de que toda base léxica comporta los tres rasgos que aparecen como nombres de tipos en la propuesta de Halliday. Todo verbo potencialmente tiene tres rasgos con polaridad más o menos positiva o más o menos negativa. Ellos son \{+/material $\},\{+/$ - mental-sensorial $\}$ y $\{+/$-relacional $\}$. La combinación es gradual y se postula en términos jerárquicos. Por lo tanto, se pueden establecer tres zonas semánticas: en la primera domina el rasgo $\{+$ material $\}$; en la segunda domina el rasgo $\{+/-$ mental-sensorial $\}$; en la tercera domina el rasgo $\{+/$-relacional $\}$. Esquemáticamente:

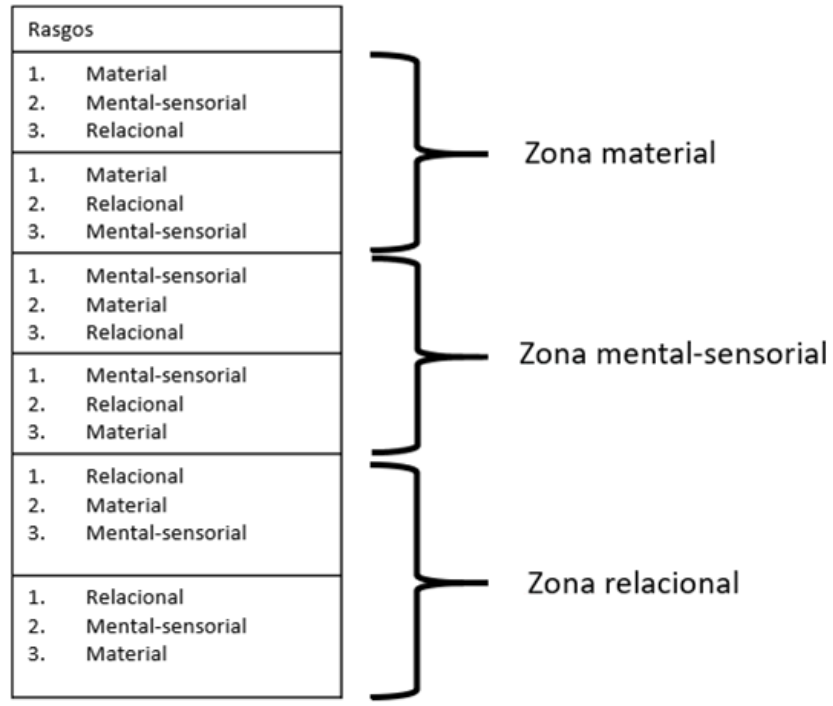

Cuadro 1. Combinación idealizada de rasgos. 
Estas tres zonas, en función de las posibilidades efectivas de realización textodiscursiva quedan reducidas a las mismas zonas con los siguientes rasgos:

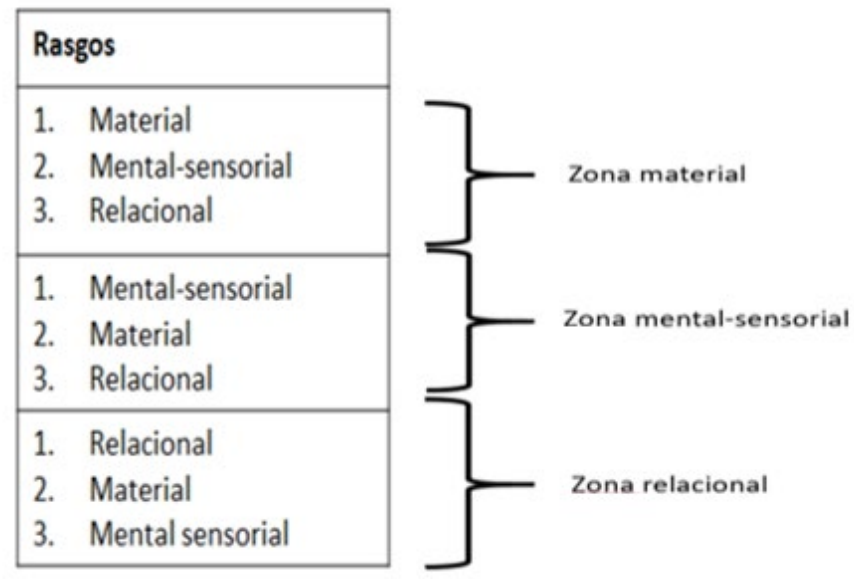

Cuadro 2. Combinación efectiva de rasgos.

En consecuencia, la gramática provee los rasgos y sus potenciales combinaciones; en el discurso, el sujeto discursivo (ver apartado 1.3.2) los realiza de manera efectiva, es decir, organiza, en función de sus necesidades, la jerarquía de rasgos al optar por un proceso particular (un recurso) como elemento puntual dentro de la estrategia que elige para expresarse.

\subsection{El grado de concreción}

La concreción supone el grado de posibilidad de que el rasgo tenga un efecto sobre un objeto materialmente asible, manipulable. El grado de concreción del proceso se maneja en una escala que va en sus extremos del 1 (+concreto) a 3 (-concreto) y depende de la organización de los rasgos en las tres zonas semánticas previamente delimitadas.

Tenemos, entonces una zona 1 en la que los aspectos materiales del proceso se hace evidentes; una zona 2, en la que los aspectos mentales-sensoriales cobran mayor importancia; por último, una zona 3 , en la que los aspectos más abstractos se hacen efectivos. Esquemáticamente: 


\begin{tabular}{|l|l|l|}
\hline Zonas & Rasgos & $\begin{array}{l}\text { Grado de } \\
\text { concreción }\end{array}$ \\
\hline 1. Material & $\begin{array}{l}\text { 1. Material } \\
\text { 2. Mental-sensorial } \\
\text { 3. Relacional }\end{array}$ & 1. [+concreto] \\
\hline 2. Mental-sensorial & $\begin{array}{l}\text { 1. Mental-sensorial } \\
\text { 2. Material } \\
\text { 3. Relacional }\end{array}$ & 2. [+/- concreto] \\
\hline 3. Relacional & $\begin{array}{l}\text { 1. Relacional } \\
\text { 2. Material } \\
\text { 3. Mental sensorial }\end{array}$ & 3. [- concreto] \\
\hline
\end{tabular}

Cuadro 3. Grados de concreción.

\subsection{La agentividad}

La tercera variable por considerar es la agentividad. Una serie de precisiones deben hacerse al respecto. La categoría de agentividad está ligada, en la propuesta de la GSF, fuertemente a la interpretación alternativa y complementaria de la transitividad: la ergatividad. El sistema de transitividad reconoce diferentes tipos de procesos con sus roles inherentes específicos. Los procesos materiales exigen un actor; los mentalessensoriales, un procesador; los relacionales, un rol inherente atributivo o identificador; los de conducta, un conductor; los de decir, un dicente y los existenciales, un existente. La interpretación ergativa (Halliday \& Matthiessen, 2014) tiene un alcance más general y apunta al origen o causa de los procesos o su agente y si quien los origina es quien los ejecuta de manera efectiva.

Una primera aproximación nos permite entonces tomar la agentividad como una propiedad gramatical que conlleva dos rasgos [+/- causa; +/-ejecucion]. Puede combinar los dos rasgos de la agentividad: ser la causa y la ejecutora del significado ideativo del proceso o ser la causa, pero no la ejecutora de ese significado. 
Esto nos permitirá agregar a nuestro cuadro 3 otra columna que lo complementa.

\begin{tabular}{|c|c|c|c|c|}
\hline \multicolumn{2}{|c|}{ Rasgos } & \multirow{2}{*}{$\begin{array}{l}\begin{array}{l}\text { Grado de } \\
\text { concreción }\end{array} \\
1 \text { [+concreto] }\end{array}$} & \multirow{2}{*}{$\begin{array}{l}\text { Agentividad } \\
\text { No causa pero } \\
\text { ejecuta la naturaleza } \\
\text { semántica del } \\
\text { proceso; }\end{array}$} & \\
\hline $\begin{array}{l}1 . \\
2 . \\
3 .\end{array}$ & $\begin{array}{l}\text { Material } \\
\text { Mental- } \\
\text { sensorial } \\
\text { Relacional }\end{array}$ & & & $\begin{array}{c}\text { Zona } \\
\text { material }\end{array}$ \\
\hline $\begin{array}{l}1 . \\
2 . \\
3 .\end{array}$ & $\begin{array}{l}\text { Mental- } \\
\text { sensorial } \\
\text { Material } \\
\text { Relacional }\end{array}$ & $2[+/-$ concreto $]$ & $\begin{array}{l}\text { Causa y/o ejecuta la } \\
\text { naturaleza } \\
\quad \text { semántica del } \\
\text { proceso }\end{array}$ & $\begin{array}{c}\text { Zona mental- } \\
\text { sensorial }\end{array}$ \\
\hline & $\begin{array}{l}\text { Relacional } \\
\text { Material } \\
\text { Mental- } \\
\text { sensorial }\end{array}$ & 3 [- concreto] & $\begin{array}{l}\text { No causa ni ejecuta } \\
\text { la naturaleza } \\
\text { semántica del } \\
\text { proceso }\end{array}$ & $\begin{array}{c}\text { Zona } \\
\text { relacional }\end{array}$ \\
\hline
\end{tabular}

Cuadro 4. Zonas en función de rasgos, grados de concreción y agentividad.

La amplitud del alcance de la agentividad nos lleva a un replanteo y reconsideración de las características de la categoría. Nos interesa ver en función de lo que venimos sosteniendo que la agentividad debe considerarse en dos planos interdependientes: el gramatical y el discursivo. Esto supone una serie de aclaraciones. La primera es disntinguir sujeto textual de sujeto discursivo. El primero subsume los sujetos de las tres funciones del lenguaje que se proyectan en la cláusula, es decir, el lógico, el interpersonal y el psicológico que, a su vez, se proyectan en el sujeto del texto que es, desde nuestro punto de vista, el sujeto de la unidad gramatical máxima que la LSF propone.

\subsubsection{La agentividad textual (ideativa)}

El sujeto textual tiene tres dimensiones complementarias que se realizan como sujeto lógico, interpersonal y textual. Si bien el lógico es el centro, en principio, de nuestra atención, no debe olvidarse la presencia de las otras dos dimensiones. El sujeto textual, entonces, permite organizar gramaticalmente el grado de concreción de los procesos con su jerarquía asociada de rasgos. La gramática, entonces, provee la virtualidad de las opciones y sus combinaciones posibles. Esto significa que, en la dimensión potencial, que se realiza paradigmáticamente, el sujeto lógico más esperable es el que tiene como rasgo preponderante el primero en la zona que le corresponde. Si tenemos un verbo inscripto en la zona material, este tendrá como rasgo número 1 el material, el grado de concreción 1 y no será causa pero si ejecutor de la naturaleza semántica del proceso. Esquemáticamente: 


\begin{tabular}{|c|c|c|c|c|}
\hline Rasgos & Grado de & Agentividad & & \\
\hline $\begin{array}{ll}\text { 1. } & \text { Material } \\
\text { 2. } & \text { Mental- } \\
\text { sensorial } \\
\text { 3. } & \text { Relacional }\end{array}$ & 1 [+concreto] & $\begin{array}{l}\text { No causa pero } \\
\text { ejecuta la naturaleza } \\
\text { semántica del } \\
\text { proceso; }\end{array}$ & $\begin{array}{c}\text { Zona } \\
\text { material }\end{array}$ & \\
\hline $\begin{array}{ll}\text { 1. } & \text { Mental- } \\
\text { 2ensorial } \\
\text { 2. Material } \\
\text { 3. }\end{array}$ & $2[+/-$ concreto $]$ & $\begin{array}{l}\text { Causa y/o ejecuta la } \\
\text { naturaleza } \\
\quad \text { semántica del } \\
\text { proceso }\end{array}$ & $\begin{array}{c}\text { Zona mental- } \\
\text { sensorial }\end{array}$ & $\begin{array}{l}\text { Sujeto } \\
\text { textual }\end{array}$ \\
\hline $\begin{array}{ll}\text { 1. } & \text { Relacional } \\
\text { 2. } & \text { Material } \\
\text { 3. } & \text { Mental- } \\
& \text { sensorial }\end{array}$ & 3 [- concreto] & $\begin{array}{l}\text { No causa ni ejecuta } \\
\text { la naturaleza } \\
\text { semántica del } \\
\text { proceso }\end{array}$ & $\begin{array}{c}\text { Zona } \\
\text { relacional }\end{array}$ & \\
\hline
\end{tabular}

Cuadro 5. Alcance del sujeto textual.

Tomemos el siguiente ejemplo: "Juan pateó la pelota". Este texto potencial para poder ser analizado e interpretado debe necesariamente contextualizarse (esto supondría un pasaje a la dimensión discursiva, ver apartado 1.3.2.). Dos contextos aparecen como posibles. En el primero, Juan va caminando por una calle en la un grupo de personas juega a la pelota. Algún jugador patea la pelota fuera del perímetro de juego y Juan recibe esa pelota y la patea instintivamente. En el segundo, Juan está jugando al futbol como miembro de un equipo dentro de una cancha. En la primera situación, el verbo 'patear' sería un verbo de la zona 1 con los rasgos, el grado de concreción y de agentividad correspondientes. En la segunda situación, un verbo de zona 2.

Por lo tanto, el pasaje del sujeto textual al discursivo es el pasaje de la virtualidad de la opción paradigmática a la instanciación de la realización sintagmática y, en consecuencia, al discurso efectivo producido por un sujeto en una situación particular.

Este sujeto textual se realiza discursivamente por el sujeto discursivo. Y es este sujeto discursivo el que manipula, evalúa y decide sobre la organización concreta de los rasgos produciendo desplazamientos semánticos, metaforizando el alcance original de la propuesta gramatical. Ahí aparece la mutua interdependencia entre gramática y discurso que no es sino la interacción entre lo potencial y lo realizado a partir de un sujeto posible y de un sujeto que se conforma y se constituye en la producción discursiva concreta.

Una aclaración aquí es pertinente: el texto es una unidad semántico-gramatical en el que se proyectan los distintos recursos que proveen los tres sistemas que representan las tres funciones del lenguaje. Esta precisión es importante ya que hacemos un distinción teórico-metodológica entre texto y discurso. El texto es una unidad semántico-gramatical que nos permite dar cuenta del sistema representado por las opciones paradigmáticas disponibles y su realización en forma de recurso en la estructura, es decir, en la realización concreta que supone la opción paradigmática 
efectivamente realizada como recurso gramatical. Una vez que ese recurso gramatical se combina con otros recursos gramaticales y/o pragmáticos estamos ante la presencia del discurso. La razón de esto es simple: hay un sujeto discursivo que produce esa combinación a partir de una estrategia que es el objeto del análisis que llevamos a cabo.

\subsubsection{La agentividad discursiva}

Como hemos visto, la gramática provee una jerarquización potencial de rasgos, de grados de concreción y agentividad. En el discurso, esto puede cambiarse y se producen desplazamientos semánticos que tienen un responsable efectivo: el sujeto discursivo. ¿Cuál es la función de este sujeto? La de combinar esos rasgos produciendo, muchas veces, diferentes tipos de desplazamientos semánticos que tienen efectos interpretativos.

El sujeto discursivo es el encargado de organizar la jerarquía de rasgos a partir de producir o no desplazamientos semánticos. Estos se representan a partir de la reorganización de los rasgos en función de la estrategia discursiva que está llevando a cabo. Este desplazamiento es siempre gradual y se expresa en términos la organización de los rasgos, el grado de concreción y la agentividad. En nuestro ejemplo anterior y a partir del cuadro 6 podemos mostrar ese desplazamiento de la siguiente manera:

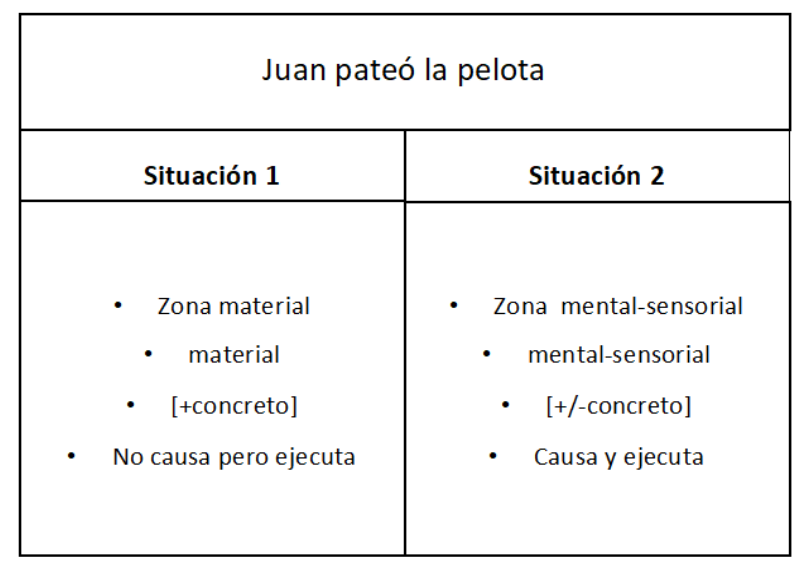

Cuadro 6. Situaciones y asignaciones de rasgos.

El recurso es el elemento de base que compone una estrategia discursiva. Esta se compone de un conjunto de ellos para poder explicar su funcionamiento e interpretar su alcance. El recurso actúa, entonces, como la opción gramatical efectivamente realizada en el discurso por el sujeto que la combina con las otras disponibles para su finalidad interaccional. Esquemáticamente: 


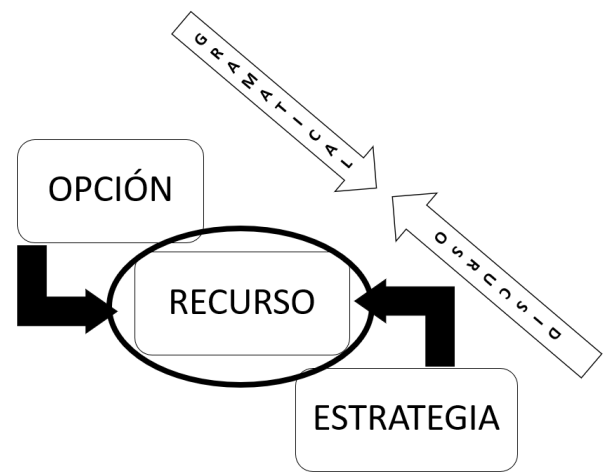

Figura 2. Recurso: Opción potencial, estrategia realizada.

Las variables analizadas nos permiten dar cuenta de cómo analizar un recurso gramatical y proyectarlo discursivamente. Los elementos que provee la gramática son el grado de concreción de la base léxica del proceso, los rasgos que permiten dar cuenta de ella y la agentividad gramatical que supone. La proyección discursiva toma estos elementos y puede o no desplazarlos semánticamente. Eso permitirá explicar el funcionamiento de estas bases léxicas e interpretar discursivamente el alcance que ese desplazamiento produce. Esquemáticamente:

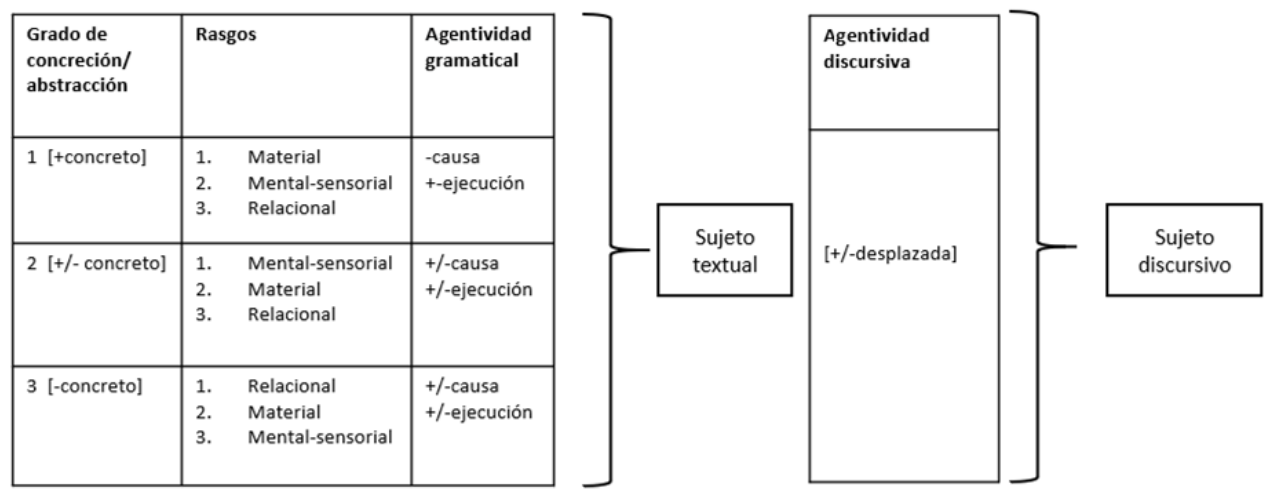

Cuadro 7. Sujeto discursivo: Alcance.

\section{Un ejemplo}

Analizaremos un ejemplo a partir de una serie discursiva con un movimiento específico dentro de una estrategia discursiva general que denominamos: "Titular una noticia". La serie está compuesta por los titulares de las páginas web los diarios de circulación nacional en la Argentina del lunes 1 de agosto de 2016 La Nación, Página12, Clarín, Infobae, Crónica y Perfil sobre un mismo hecho: las opiniones que el papa Francisco dio sobre los índices de desocupación en la Argentina. Nos concentramos 
exclusivamente aquí en el análisis de los procesos para poder determinar el alcance y necesaria complementación entre la agentividad gramatical (textual) y la discursiva.

\subsection{Corpus}

Transcribimos los titulares:

\begin{tabular}{|l|l|l|}
\hline Nro. & Diario & \multicolumn{1}{c|}{ Titular } \\
\hline 1 & Clarín & El Papa advierte que el desempleo en el país es "significativamente alto" \\
\hline 2 & Crónica & Francisco le pidió a San Cayetano más "pan y trabajo" para los argentinos " \\
\hline 3 & Infobae & $\begin{array}{l}\text { Los índices de desocupación son significativamente altos en Argentina", dijo el } \\
\text { Papa }\end{array}$ \\
\hline 4 & $\begin{array}{l}\text { La } \\
\text { Nación }\end{array}$ & $\begin{array}{l}\text { El papa Francisco denuncia en una carta los altos índices de desocupación en la } \\
\text { Argentina }\end{array}$ \\
\hline 5 & Página 12 & El Papa ve "índices de desocupación significativamente altos" \\
\hline 6 & Perfil & El Papa Francisco afirma que en el país el desempleo es "significativamente alto" \\
\hline
\end{tabular}

\subsection{Análisis}

De acuerdo con nuestro planteo, se deben tomar en consideración cuatro variables, tres gramaticales y una discursiva, descriptas anteriormente. Son:

1. el grado de concreción del proceso,

2. el orden jerárquico de los rasgos,

3. la relación entre causa y ejecución

4. el grado de desplazamiento

Las opciones léxicas pertenecen a los verbos de la cláusula de los titulares que presentan la información que en ellos se detallan. Los que aparecen en las subordinadas (como en 1, 3 y 5) no se consideran en función de que no son representativas para el análisis que estamos llevando a cabo.

\begin{tabular}{|l|l|}
\hline Nro & Verbo \\
\hline 1 & Advierte \\
\hline 2 & Pidió \\
\hline 3 & Dijo \\
\hline 4 & Denuncia \\
\hline 5 & Ve \\
\hline 6 & Afirma \\
\hline
\end{tabular}

Analizamos las seis bases verbales de los procesos a partir de los propuesto en 1.1., 1.2. y 1.3. En la serie aparecen seis bases léxicas verbales que se ubican dentro del grado de concreción 2; esto quiere decir que activan rasgos mentales y materiales con preeminencia del primer rasgo sobre el segundo: hay un grado de conciencia efectivo de quien llevará a cabo el significado que la base léxica describe; hay, por lo tanto, un procesamiento por parte del sujeto ideativo que se representa en la causalidad y ejecución de lo que la base léxica significa. La zona mental-sensorial cubre los verbos 
en los que la agentividad del sujeto textual aparece siempre como una necesidad evidente más allá del grado de materialidad de aquello sobre lo que la agentividad actúa.

\begin{tabular}{|c|c|c|c|c|}
\hline Nro & Verbo & $\begin{array}{l}\text { Grado de } \\
\text { concreción }\end{array}$ & $\begin{array}{c}\text { Rasgos } \\
\text { MS }=\text { mental }- \text { sensorial } \\
\text { M=material } \\
\mathbf{R}=\text { relacional }\end{array}$ & $\begin{array}{c}\text { Agentividad gramatical } \\
\begin{array}{c}\mathrm{C}=\text { causa } \\
\mathrm{E}=\text { ejecuta }\end{array}\end{array}$ \\
\hline 1 & Advierte & 2 & MS, M, R & $+/-\mathrm{C},+/-\mathrm{E}$ \\
\hline 2 & Pidió & 2 & MS, M, R & $+/-\mathrm{C},+/-\mathrm{E}$ \\
\hline 3 & Dijo & 2 & $\mathrm{MS}, \mathrm{M}, \mathrm{R}$ & $+/-\mathrm{C},+/-\mathrm{E}$ \\
\hline 4 & Denuncia & 2 & $\mathrm{MS}, \mathrm{M}, \mathrm{R}$ & $+/-\mathrm{C},+/-\mathrm{E}$ \\
\hline 5 & $\mathrm{Ve}$ & 2 & MS. M R. & $+/-\mathrm{C},+/-\mathrm{E}$ \\
\hline 6 & Afirma & 2 & MS, M, R & $+/-\mathrm{C},+/-\mathrm{E}$ \\
\hline
\end{tabular}

De acuerdo con lo que observamos en la tabla anterior, desde el punto de vista de la gramática, los verbos suponen una caracterización similar. Los rasgos que suponen la elección de cada uno de ellos deben explicarse a partir de su inscripción discursiva. Es en la realización discursiva concreta en la que las especificaciones semánticogramaticales de cada uno de ellos puede establecerse. La agentividad discursiva representa la acción que lleva a cabo el sujeto discursivo. Este produce un grado de desplazamiento en la selección particular de cada una de esas bases léxicas. La consecuencia es evidente: a menor grado de desplazamiento, hay coincidencia entre sujeto gramatical y discursivo; a mayor grado, menor es esa coincidencia. Esto es fundamental porque la polaridad que adquieren los rasgos de la agentividad gramatical es asignada justamente por el sujeto discursivo. Este sujeto produce un texto: de él depende la selección del grado de coincidencia o desplazamiento con las bases léxicas. Allí se puede comenzar a medir el efecto de interpretación deseado dentro del esquema estratégico general en el que este recurso se ubica.

Transcribimos el cuadro con una nueva columna, que marca el grado de desplazamiento operado por el sujeto discursivo sobre la agentividad gramatical. La potencialidad de los rasgos de la agentividad gramatical que aparecen en el cuadro anterior aparece modificado en función de la acción de la agentividad discursiva.

\begin{tabular}{|c|c|c|c|c|c|}
\hline Nro & Verbo & $\begin{array}{c}\text { Grado de } \\
\text { concreción }\end{array}$ & Rasgos & $\begin{array}{c}\text { Agentividad } \\
\text { gramatical }\end{array}$ & $\begin{array}{c}\text { Agentividad } \\
\text { discursiva }\end{array}$ \\
\hline 6 & Afirma & 2 & MS, M, R & $+\mathrm{C},+\mathrm{E}$ & -desplazado \\
\hline 3 & Dijo & 2 & MS, M, R & $+\mathrm{C},+\mathrm{E}$ & -desplazado \\
\hline 1 & Advierte & 2 & MS, M, R & $-\mathrm{C},+\mathrm{E}$ & + desplazado \\
\hline 2 & Pidió & 2 & MS, M, R & $-\mathrm{C},+\mathrm{E}$ & + desplazado \\
\hline 4 & Denuncia & 2 & MS, M, R & $-\mathrm{C},+\mathrm{E}$ & + desplazado \\
\hline 5 & Ve & 2 & MS. M R. & $-\mathrm{C},+\mathrm{E}$ & +desplazado \\
\hline
\end{tabular}

De los seis verbos dos operan sin desplazamiento semántico (decir, afirmar) y el resto con desplazamiento semántico (advertir, pedir, denunciar, ver) con un grado 
mayor. La justificación de estos desplazamientos debe buscarse en la proyección que hace el sujeto discursivo sobre el textual. Si hay coincidencia en la dimensión del alcance interpretativo de ambos, el grado coincide; si no la hay, el discursivo actúa sobre el primero operando el desplazamiento. Eso se observa en el hecho de que en la agentividad gramatical se produce un cambio de polaridad en el rasgo [+causal]. Un principio de orden general tentativo es: a menor desplazamiento de la agentividad discursiva, mayor causalidad en la agentividad gramatical.

En los dos primeros casos de nuestro corpus (números 3 y 6) los agentes discursivos y los gramaticales coinciden y el sujeto discursivo no produce ninguna modificación. Los verbos 'decir' y 'afirmar' marcan un grado de coincidencia entre el sujeto discursivo (el medio que produce el titular) y el sujeto textual (el texto que ese medio produce).

Los otros cuatro verbos muestran cómo el sujeto discursivo toma una posición evaluativa y orienta el efecto interpretativo del titular al asignarle al sujeto textual una dimensión semántico-discursiva que no es sino su proyección. 'Advertir', 'Pedir', 'Denunciar' y 'Ver' son interpretaciones del sujeto discursivo que provocan un desplazamiento semántico-discursivo. Se justifica gramaticalmente a partir de que los cuatro tienen marcas de polaridad negativa en el rasgo [causa] de su agentividad gramatical. Al tener este rasgo con esa polaridad, el sujeto textual ejecuta algo que no causa.

En el caso de 'Pedir' podría pensarse como una coincidencia entre ambos sujetos (como es el caso 3 y 6) puesto que quien, supuestamente, lleva a cabo la petición es el sujeto textual (el Papa). Pero, sostenemos, que hay un desplazamiento ya que el sujeto textual interpreta que el Papa hizo un pedido cuyo objeto (pan y trabajo) aparece entrecomillado. No hay cita directa y no nos consta que lo haya hecho en esos términos. La consecuencia es evidente: quien asigna la orientación de los efectos de interpretación del discurso no es el agente de la acción discursiva original (el Papa, en este caso) sino el medio que le atribuye esa acción. Esto permite producir distintos efectos que tienen que ser enmarcados dentro del esquema general estratégico del que este análisis toma solamente un recurso puntual.

En nuestro ejemplo, los cuatro desplazamientos obedecen a evaluaciones del sujeto discursivo sobre el tema que está tratando. Los alcances de su selección siempre son estratégicos y deben analizarse como tales. Y el grado de desplazamiento semántico es un elemento puntual y concreto para poder marcar los alcances de ese desplazamiento. Dentro de él pueden operar procedimientos de orden metafórico que no serían sino el grado alto de desplazamiento (caso número 5 de nuestro corpus).

Si retomamos nuestro corpus y analizamos solamente este recurso, podríamos intentar una primera caracterización (que luego deberá complementarse con las relaciones que este recurso tiene con los otros recursos, tanto gramaticales como 
discursivos, dentro la perspectiva estratégica adoptada) y proponer tentativamente un orden de desplazamiento, en función de la proyección textual del proceso en la dimensión discursiva.

La selección de los verbos 'decir' y 'afirmar', en estos titulares, tiene un grado de desplazamiento bajo en función de la coincidencia del sujeto textual y discursivo en términos de causa y ejecución de contenido semántico. Esa coincidencia no se produce del mismo modo en 'advertir', 'pedir' y 'denunciar' ya que allí el sujeto discursivo evalúa. Sucede lo mismo, pero con un desplazamiento metafórico evidente en 've' cuyo rasgo de materialidad de base semántica evidente permite analizar el tipo de evaluación que se está llevando a cabo. En consecuencia, en esta serie puntual, el agente gramatical (el Papa) 'afirma' y 'dice'. Decir o afirmar algo no es ni advertir, ni pedir, ni denunciar ni ver algo.

Podemos, por lo tanto, tentativamente agrupar los grados de desplazamiento del sujeto discursivo en relación con el sujeto textual en tres subgrupos de acuerdo con el grado de desplazamiento.

\begin{tabular}{|c|c|c|}
\hline Verbo & Agentividad gramatical & Agentividad discursiva \\
\hline Decir & $+\mathrm{C},+\mathrm{E}$ & -desplazado \\
\hline Afirmar & $+\mathrm{C},+\mathrm{E}$ & -desplazado \\
\hline Advertir & $-\mathrm{C},+\mathrm{E}$ & + desplazado \\
\hline Pedir & $-\mathrm{C},+\mathrm{E}$ & + desplazado \\
\hline Denunciar & $-\mathrm{C},+\mathrm{E}$ & + desplazado \\
\hline Ve & $-\mathrm{C},+\mathrm{E}$ & ++ desplazado (metáfora) \\
\hline
\end{tabular}

El grado de desplazamiento está en relación con el posicionamiento que el sujeto discursivo adopta en su selección de recursos que adopta para manejarse estratégicamente. Sin embargo, debe precisarse que el análisis del recurso en sí mismo es válido pero limitado. La razón de esto es simple: el objetivo que perseguimos es justamente su explicación en función de cómo debe ser analizado dentro de una estrategia discursiva.

\section{CONCLUSIONES}

Hemos planteado que la proyección discursiva de los procesos se debe llevar a cabo a partir de una caracterización de una gradualidad semántica que permite establecer rasgos que se combinan con grados de concreción, agentividad textual y discursiva.

La agentividad discursiva permite establecer si el agente es causante y/o ejecutor del contenido semántico que la base léxica del proceso comporta. Sostenemos, entonces, que el sujeto ideativo debe inscribirse dentro del sujeto textual como uno de sus elementos constitutivos. Quedan para futuros desarrollos la relación entre el sujeto textual con el sujeto gramatical y el sujeto temático porque son esas tres dimensiones 
del sujeto textual las que se proyectan en el discurso a partir de la organización que el sujeto discursivo al producir su discurso le impone. Es importante, entonces, tener en cuenta que el planteo llevado a cabo está orientado hacia el análisis estratégico de los discursos y lo analizado es simplemente un recurso que debe complementarse a partir de las relaciones que establece con otros recursos gramaticales (que proveen el sistema de modo, el de tema y las relaciones cohesivas) y semántico-discursivo o pragmáticos (la ilocución y los dispositivos suposicionales y evaluativos) (Menéndez, 2019).

Mostramos que la gramática provee rasgos, pero es el sujeto discursivo el que lleva a cabo su activación efectiva a partir de su jerarquización en función de las necesidades interaccionales que persigue. La descripción de los rasgos permite su explicación en función del desplazamiento que el sujeto discursivo opera en ellos permitiendo, de esa manera, posibilidades de interpretación que están siempre condicionadas por los supuestos, el registro y el género, es decir, por el contexto (tanto en su dimensión socio-cognitiva como en la socio-cultural).

\section{REFERENCIAS BIBLIOGRÁFICAS}

Bajtin, M. (1984). El problema de los géneros discursivos. En M. Bajtin (Ed.), Estética de la creación verbal (pp. 248-294). México: Siglo XXI.

Fillmore, Ch. (1968). The case for case. En E. Bach \& R. Harms (Eds.), Universals in Linguistic Theory (pp. 1-90). Nueva York: Holt, Rinehart and Winston.

Halliday, M. A. K. (1967a). Notes on transitivity and theme in English (Part 1). Journal of Linguistics, 3(1), 37-81.

Halliday, M. A. K. (1967b). Notes on transitivity and theme in English (Part 2). Journal of Linguistics, 3(2), 199-244.

Halliday, M. A. K. (1968). Notes on transitivity and theme in English (Part 3). Journal of Linguistics, 4(2), 179-215.

Halliday, M. A. K. (1977). Text as semantic choice in social contexts. En T. van Dijk \& J. S. Petöfi (Eds.), Grammars and Descriptions (pp.176-225). Berlín: Mouton de Gruyter.

Halliday, M. A. K. (1978). Language as social semiotics. Londres: Edward Arnold.

Halliday, M. A. K. (1985). Introduction to functional grammar. Londres: Edward Arnold.

Halliday, M. A. K. (2013). Putting linguistic theory to work. En J. J. Webster (Ed.), The Collected Works of M. A. K. Halliday, Volume 11: Halliday in the 21st Century (pp.124-142). Londres: Bloomsbury.

Halliday, M. A. K. \& Hasan, R. (1976). Cohesion in English. Londres: Longman. 
Halliday, M. A. K. \& Matthiessen, Ch. (1999). Construing experience through meaning. Londres: Edward Arnold.

Halliday, M. A. K. \& Matthiessen, Ch. (2014). An Introduction to the Functional Grammar. Londres: Edward Arnold.

Hasan, R. (1987). The grammarian's dream: lexis as most delicate grammar. En M.A.K. Halliday y R. P. Fawcett (Eds.). New developments in systemic linguistics. Volume 1: Theory and description (pp. 184-211). London: Pinter.

Lavandera, B. (1985). Curso de lingüística para el análisis del discurso. Buenos Aires: Centro Editor de América Latina.

Lavandera, B. (1988). El lenguaje en su contexto socio-cultural. En F. Newmeyer (Ed.), Panorama de la lingüística moderna de la Universidad de Cambridge Volumen IV: Lenguaje: Contexto sociocultural (pp.15-29). Madrid: Visor.

Lavandera, B. (2014). Variación y significado. Y discurso. Buenos Aires: Paidós.

Martin, J. (1992). English text: System and structure. Ámsterdam: John Benjamins.

Martin, J. (1984). Language, register and genre. En C. Frances (Ed.), Children writing: Reader (pp. 21-30). Geelong, Vic.: Deaking University Press.

Martin, J. (1997). Analysing genre: Functional parameters. En F. Christie \& J. Martin (Eds.), Genre and institutions (pp. 3-39). Londres: Continuum.

Martin, J. (2012). A context for genre: Modelling social processes in functional linguistics. En Z. Wang (Ed.), Genre Studies: Collected Workes of J. R. Martin, Volume 3. (pp. 248-77). Shanghai: Shanghai Jiaotong University Press.

Martin, J. (2013). Systemic Functional Grammar: A next step into the theory - axial relations. Beijing: Higher Education Press.

Martin, J. \& Rose, D. (2008). Genre relations: Mapping culture. Sheffield: Equinox.

Matthiessen, C. M. I. M. (2015). Halliday's conception of language as a probabilistic System. En J. J. Webster (Ed.), The Bloomsbury Companion to M. A. K. Halliday (pp. 203-41). Londres: Bloomsbury.

Menéndez, S. M. (2000). Estrategias discursivas: Principio metodológico para el análisis pragmático del discurso. En J. J. de Bustos \& P. Chareadeau (Eds.), Lengua, discurso, texto (I Simposio Internacional de Análisis del discurso) (pp. 926945). Madrid: Visor. 
Menéndez, S. M. (2005a). Gramática, análisis del discurso e interpretación crítica: Las relaciones no tan evidentes. Proceedings of the Internacional Conference on Critical Discourse Analysis. Valencia: Universitat de Valencia. (Edición en CD).

Menéndez, S. M. (2005b). ¿Qué es una estrategia discursiva? En S. Santos \& J. Panesi (Comp.), Actas del Congreso Internacional: Debates Actuales. Las teorias críticas de la literatura y la lingüistica. Buenos Aires, Facultad de Filosofía y Letras, UBA. (Edición en CD).

Menéndez, S. M. (2006). ¿Qué es una gramática textual? Buenos Aires: Litterae Ediciones.

Menéndez, S. M. (2010). Opción, registro y contexto. El concepto de significado en la lingüística sistémico-funcional. Tópicos del seminario, 23(1), 221-240.

Menéndez, S. M. (2011a). Procesos, gradualidad, registro y género: Interdependencias necesarias. Ponencia presentada en el Coloquio Internacional de Lingüística Iberorrománica, K.U, Leuven.

Menéndez, S. M. (2011b). Processes classification: Graduality, features and agentivity. Ponencia presentada en el 38th International Systemic Functional Congress - ISFC38 Negotiating difference: Languages, metalanguages, modalities, cultures. Facultad de Letras, Universidad de Lisboa, Lisboa, Portugal.

Menéndez, S. M. (2013). La clasificación de los verbos en español: El principio de gradualidad. Un enfoque sistémico-funcional. En L. Funes (Ed.), XVIII Actas del Congreso de la Asociación Internacional de Hispanistas (pp. 253-269). Buenos Aires: Facultad de Filosofía y Letras.

Menéndez, S. M. (2014). Estudio preliminar. En B. Lavandera (Ed.), Variación y significado. $Y$ discurso (pp. XXI-XXXVI). Buenos Aires: Paidós. ISBN 978-950$12-2740$.

Menéndez, S. M. (2017a). Agentividad: Causalidad y Ejecución. La Proyección Discursiva de los Procesos. Ponencia presentada en la 27th ESFLC (European Systemic Functional Linguistics Conference), Universidad de Salamanca, Salamanca, España.

Menéndez, S. M. (2017b). Agentivity: Verbs classification from a discursive point of view. Ponencia presentada en el XV International Conference of the International Pragmatics Association. International Pragmatics Association, Belfast, Irlanda.

Menéndez, S. M. (2019) Entre la gramática y el género: El discurso. Un enfoque estratégico. En O. I. Londoño Zapata \& G. Olave (Eds.), Métodos de Análisis del discurso. Perspectivas argentinas (pp. 115-132). Bogotá: Ediciones de la U.

Tésniére, L. (1958). Elementos de sintaxis funcional. Madrid: Gredos. 
Verschueren, J. (1999). Understanding pragmatics. Londres: Edward Arnold.

\section{ADDENDA: Fecha: 1 de Agosto de 2016; 11.20 horas}
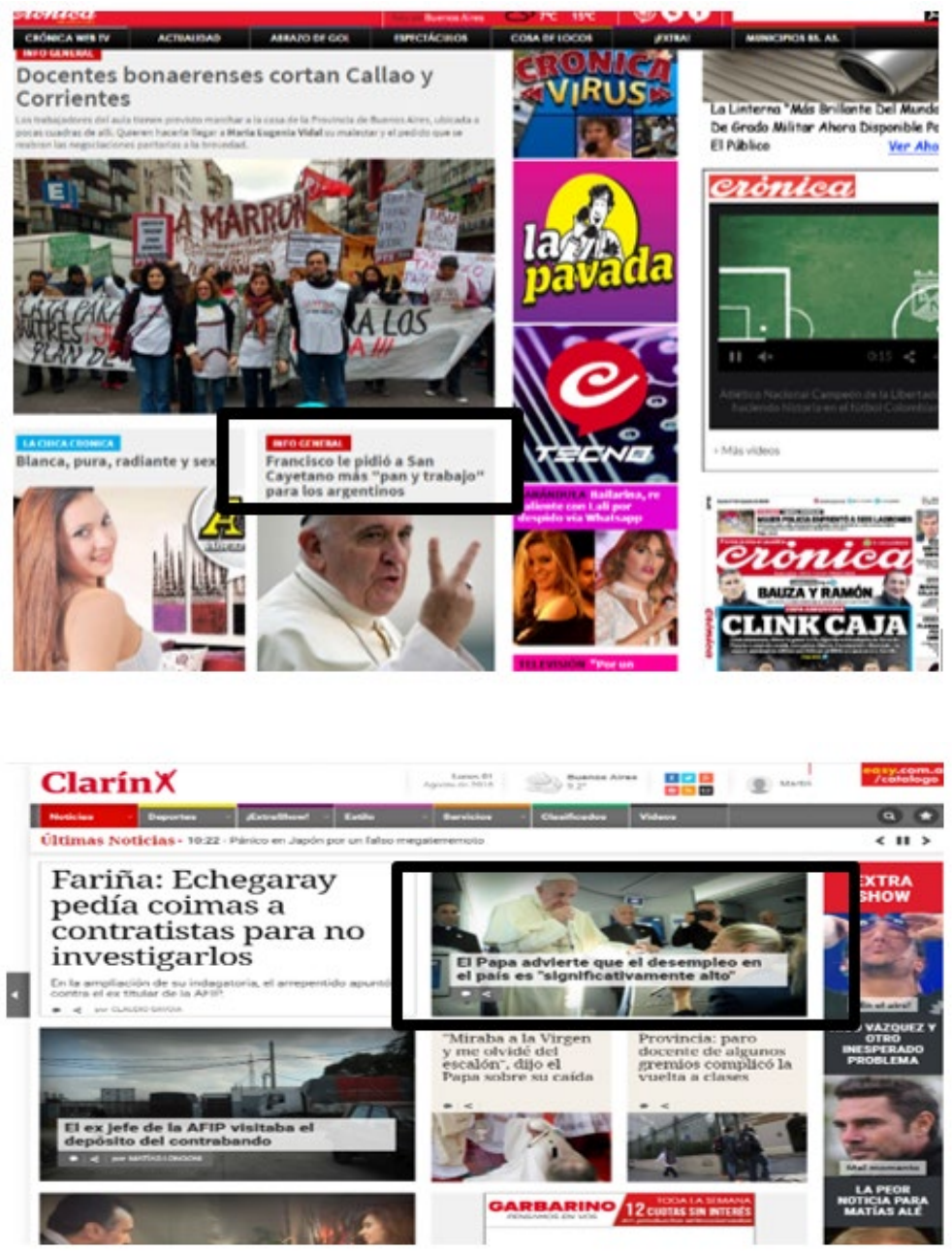


\section{LA NACION

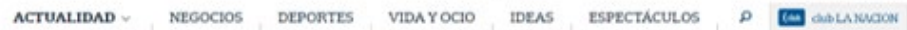

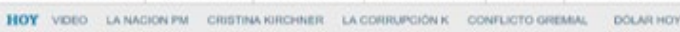

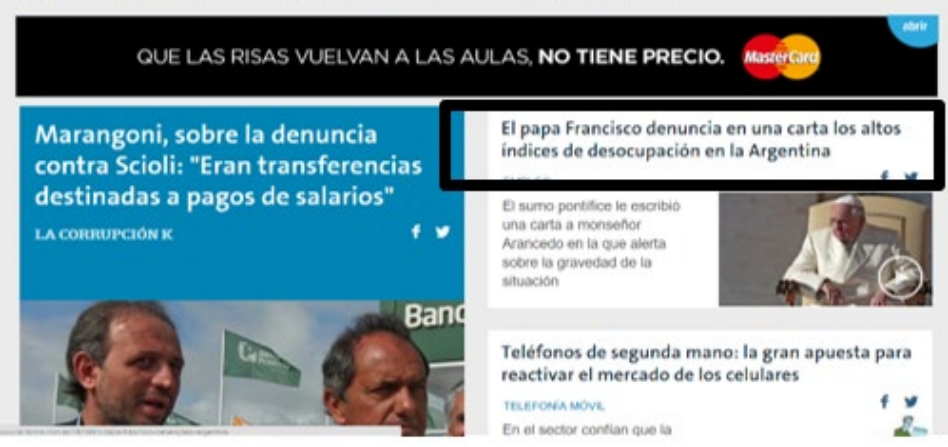

इMENu EPERFIL

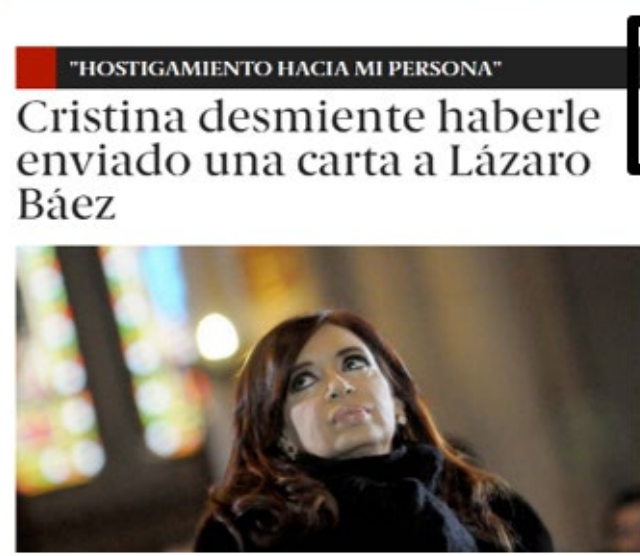

E1 Papa Francisco afirma que en el pais el

desempleo es

"significativamente alto" enviado una carta a Lázaro Báez

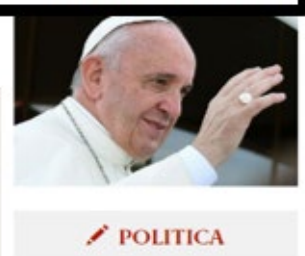

Sergio Massa y Máximo Kirchner mantuvieron un encuentro secreto Por: Recalt, Rodis

\section{Página|12}

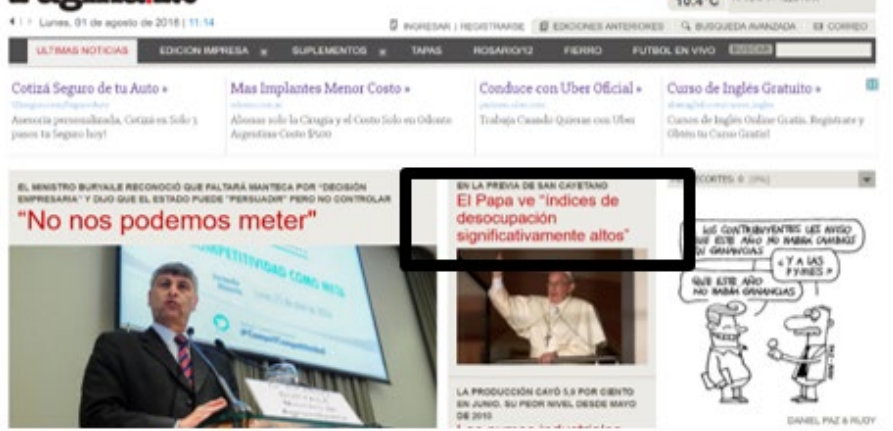




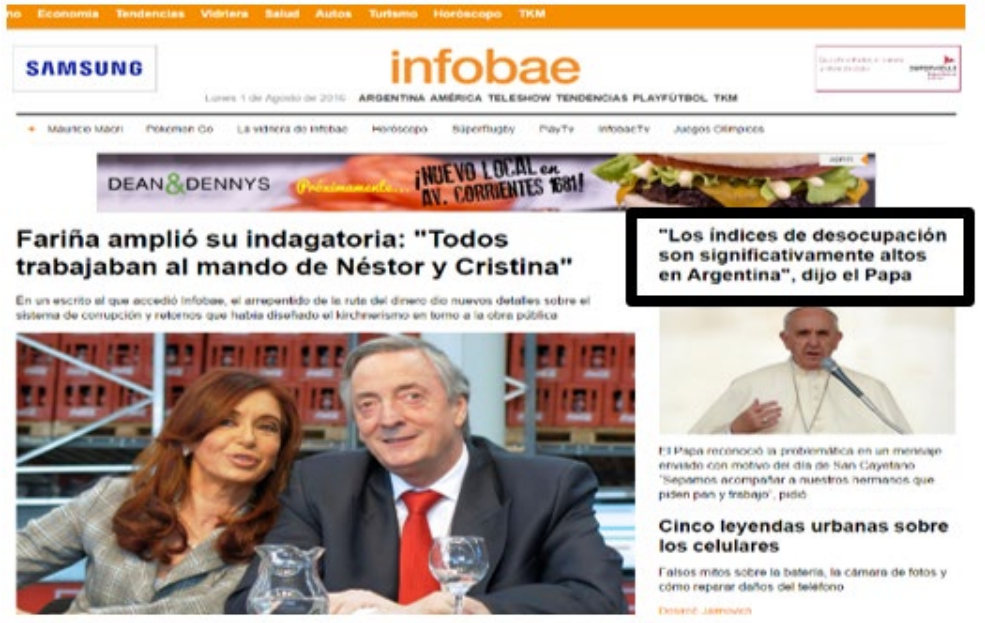

